\title{
Chromospheric Evaporation in a Remote Solar Flare-Like Transient \\ Observed at High Time Resolution With $S O H O$ 's CDS and RHESSI
}

\author{
Jeffrey W. Brosius ${ }^{1}$ \\ Gordon D. Holman ${ }^{2}$
}

\begin{abstract}
We present EUV light curves and Doppler velocity measurements for a small, remote flare-like transient observed at high time resolution $(9.8 \mathrm{~s})$ with $S O H O$ 's CDS during a GOES M1.6 solar flare. The EUV observations include a brief precursor and an impulsive peak followed by a more gradual rise and decline of emission. Hard X-ray light curves obtained with RHESSI reveal a small burst just before the EUV impulsive rise, and another burst at the time of the more gradual EUV peak. RHESSI images show no emission at the location of the EUV transient due to limitations in dynamic range. During the impulsive phase we measure simultaneous, cospatial downward velocities $\sim 30 \mathrm{~km} \mathrm{~s}^{-1}$ in the chromospheric line of $\mathrm{He} \mathrm{I}$ at $584.3 \AA$ and the transition region line of $\mathrm{O} \mathrm{V}$ at $629.7 \AA$, and upward velocities $\sim 20 \mathrm{~km} \mathrm{~s}^{-1}$ in the coronal line of Si XII at 520.7 $\AA$. Fe XIX emission at $592.2 \AA$ emerged during the impulsive phase, and revealed upward velocities approaching $150 \mathrm{~km} \mathrm{~s}^{-1}$. These observations demonstrate that flare-like explosive chromospheric evaporation occurred at a location remote from the primary region of particle acceleration, apparently driven by electron beams from the primary acceleration region.
\end{abstract}

Subject headings: Sun: activity — Sun: corona — Sun: flares — Sun: UV radiation — Sun: X-rays, gamma rays

\footnotetext{
${ }^{1}$ Catholic University of America at NASA Goddard Space Flight Center, Greenbelt, MD; brosius@helio.gsfc.nasa.gov

${ }^{2}$ NASA Goddard Space Flight Center, Greenbelt, MD; Gordon.D.Holman@nasa.gov
} 


\section{Introduction}

Neupert (1968) described the process that later became known as "chromospheric evaporation." Evaporation occurs when chromospheric material is heated by beamed particles and/or a thermal conduction front more quickly than it can radiatively cool. The heated material expands upward into the lower-density corona, and slowly downward into the higher-density chromosphere. Blueshifts in emission lines formed at temperatures $\gtrsim 10^{7} \mathrm{~K}$ during the impulsive phase of solar flares provide evidence for this phenomenon (Antonucci et al. 1999 and references therein; Bornmann 1999 and references therein; Czaykowska et al. 1999, 2001; Brosius 2003; Teriaca et al. 2003, 2006; Brosius \& Phillips 2004; Falchi et al. 2006; Milligan et al. 2006a,b). Evaporation proceeds "explosively" when lines formed in the upper chromosphere and transition region appear redshifted while hot flare lines appear blueshifted, and evaporation proceeds "gently" when lines formed in the upper chromosphere and transition region (as well as hotter lines, if present) all appear blueshifted (Fisher et al. 1985a,b,c; Brosius \& Phillips 2004; Allred et al. 2005; Milligan et al. 2006a,b). Here we present observations of a weak flare-like transient (FLT) obtained with the Coronal Diagnostic Spectrometer (CDS; Harrison et al. 1995) and the Extreme-ultraviolet Imaging Telescope (EIT; Delaboudinière et al. 1995) aboard the Solar and Heliospheric Observatory (SOHO), and coordinated observations with the Ramaty High Energy Solar Spectroscopic Imager (RHESSI; Lin et al. 2002, Hurford et al. 2002, Smith et al. 2002).

CDS provides spatially resolved, instantaneous, stigmatic EUV spectra along its 4arcmin-long slit. The instrument can be used to acquire raster images of target areas by obtaining spectra from successive slit pointings, or it can be operated in a sit-and-stare mode in which successive exposures over small (single slit) areas are obtained. Czaykowska et al. (1999, 2001), Pike \& Mason (2002), Teriaca et al. (2003, 2006), Falchi et al. (2006), and Milligan et al. (2006a,b) used CDS in its raster mode to obtain images of solar flares at cadences that range from 3.5 to 11 minutes. While all of these studies provide spectroscopic measurements of flare dynamics over limited two-dimensional fields of view, their cadence (and hence time resolution) is longer than the timescales on which impulsive phase flare emission can exhibit considerable variability.

The CDS observing sequence PBEAM was developed to obtain rapid cadence (9.83 s) spatially resolved (twelve $4^{\prime \prime} \times 20^{\prime \prime}$ slit pixels) flare spectra over 549-559 $\AA$ and 590-631 $\AA$ subsets of the CDS wavebands (Brosius 2001, 2003). High time resolution is achieved by

sacrificing spatial imaging. Brosius \& Phillips (2004) presented PBEAM observations of the commencement site (footpoint) of a flare loop before, during, and after the impulsive phase of a GOES class M2.3 flare in AR 9433 on 2001 April 24. Their measurement of upflows $\sim 40 \mathrm{~km} \mathrm{~s}^{-1}$ in the relatively cool EUV lines of O III, O IV, O V, and He II during two 
flare precursors suggested that gentle evaporation occurred at those times. The same lines exhibited downflows $\sim 40 \mathrm{~km} \mathrm{~s}^{-1}$ during the flare impulsive phase, when the hot flare line of

Fe XIX became strong and blueshifted, with a maximum upflow speed of $64 \mathrm{~km} \mathrm{~s}^{-1}$. This, along with comparable upflows measured in hot flare lines of S XV and Ca XIX observed with Yohkoh's BCS, indicated explosive evaporation. The velocities reported by Brosius \& Phillips are consistent with those derived in the hydrodynamic simulations of Fisher et al. (1985a,b,c), Emslie et al. (1992), and Allred et al. (2005).

The CDS observing sequence FLAREDOP is a modified version of PBEAM. It obtains spectra within 515-525, 580-610, and 623-631 $\AA$ wavebands at a cadence of $9.81 \mathrm{~s}$. The FLAREDOP study was run on AR $10652\left(-141^{\prime \prime},+58^{\prime \prime}\right)$ between 17:31:07 and 23:37:15 UT on 2004 July 22 during a series of four flares, including a GOES class C3.2 event (17:17 17:37 UT), a C4.1 event (22:01 - 22:18 UT), an M1.6 (22:40 - 23:07 UT), and an M1.2 (23:10 - 23:43 UT). In this Letter we report EUV spectroscopic measurements of line intensities and Doppler velocities during a small flare-like transient (FLT) that began at 22:45 UT and occurred an arcminute or more away from the flare sources observed with EIT and RHESSI. We focus on local plasma dynamics in the FLT, and find that explosive chromospheric evaporation occurs at this location remote from the primary region of particle acceleration.

\section{Observations and Results}

The CDS pointing was fixed $\left(\mathrm{x}=-141^{\prime \prime}, \mathrm{y}=+58^{\prime \prime}\right)$, so features slowly drifted across the CDS field of view due to solar rotation. Fig. 1 shows the pointing of the CDS slit during a sequence of $4^{\prime} \times 4^{\prime}$ EIT images extracted from full Sun images obtained at $195 \AA$ during our observing run. The $4^{\prime \prime} \times 240^{\prime \prime}$ CDS slit, divided into twelve $4^{\prime \prime} \times 20^{\prime \prime}$ pixels, is superimposed on each image. The image pixel size is $2^{\prime \prime} .63$, and intensities in all six frames are normalized equally. EIT typically acquires a full Sun image every 12 minutes in its $195 \AA$ waveband but, unfortunately, images at 22:48 and 23:00 UT are missing (as are TRACE observations during this same period). The $195 \AA$ waveband is nearly always dominated by emission from Fe XII over most of the solar corona, but during a flare Fe XXIV emission at $192 \AA$ is also likely to contribute; these lines are formed at $\log \mathrm{T}_{m}=6.1$ and $\log \mathrm{T}_{m}=7.3$, respectively, where the "formation temperature" $\mathrm{T}_{m}$ (in $\mathrm{K}$ ) of an emission line is the temperature that maximizes the emitting ion's fractional abundance (e.g., Mazzotta et al. 1998). EIT intensity contours are overplotted in all frames of Fig. 1 except (c); frame (c) displays contours of 20-40 keV hard X-ray (HXR) counts obtained with RHESSI. The FLT is located in the third CDS slit pixel up from the bottom, evident as a brightening in the EIT images (see arrow in Fig. 1d) but invisible in the RHESSI image due to limitations on dynamic range. 
One full set of CDS spectra was obtained every 9.81 s. These were processed and calibrated with standard SolarSoftware IDL procedures. We used the "broadened gaussian" line profile fitting procedure developed by Thompson (1999) to obtain profile fits to emission lines. Further discussion of why and how this procedure is used to obtain centroid wavelengths and integrated intensities can be found in Brosius (2003) and Brosius \& Phillips (2004). In this work we concentrate on the lines of He I $\left(\log \mathrm{T}_{m}<4.3\right)$ at $584.3 \AA$, O V $\left(\log \mathrm{T}_{m}=5.4\right)$ at $629.7 \AA$, Si XII $\left(\log \mathrm{T}_{m}=6.3\right)$ at $520.7 \AA$, and Fe XIX $\left(\log \mathrm{T}_{m}=6.9\right)$ at $592.2 \AA$. Results for these lines at the location of the FLT are displayed in Fig. 2 and 3. We also include the $\mathrm{Mg} X\left(\log \mathrm{T}_{m}=6.1\right)$ line at $624.9 \AA$, with an O IV line at $625.8 \AA$ (which we fit) in its red wing, and a weaker (but inseparable) O IV blend at $625.1 \AA$.

We examined light curves of He I, O V, Si XII, and Fe XIX from each of the 12 CDS slit spatial pixels for the entire observing period. Numerous short term flickerings, especially in $\mathrm{O}$ $\mathrm{V}$, were observed across the active region. Several gradual brightenings in Fe XIX emission were observed, likely due to loops filling with hot plasma during the GOES flares listed above. A unique feature that caught our attention in these observations, however, was one in which the Fe XIX emission rose impulsively above its background noise, remained elevated for about 18 minutes during which it showed two major peaks, and then disappeared again to its preflare noise level. This occurred in the 3rd CDS slit pixel up from the bottom in Figure 1, indicated with an arrow in frame (d) at 23:12 UT; it appears as a compact source outlined by the second EIT contour in the image at this time and location. Light curves of He I and O V reveal sharp intensity spikes (followed by a second intensity peak) in the same pixel around the same time, while that of Si XII reveals an impulsive rise followed by a slow, steady rise and decline. In Figure 2 we show light curves of He I, O V, Mg X, Si XII, and Fe XIX over a 1.1 hour time period around the FLT. For each line the pre-FLT intensity shows only a very slow increase with time, primarily due to the drift (solar rotation) of brighter areas across the fixed slit position. A noticeable increase in the Fe XIX emission, although very weak and noisy, begins around 22:43:46.6 UT. A brief precursor, which lasts about 40 s and peaks at 22:45:24.7 UT, is seen in He I, O V, and possibly Mg X. This is followed by an impulsive intensity rise, which begins at 22:45:45.0 UT and leads to spikes in the He I, O $\mathrm{V}$, and $\mathrm{Mg} \mathrm{X}$ emission. The $\mathrm{O} \mathrm{V}$ and $\mathrm{Mg} \mathrm{X}$ emission peaks at 22:46:43.2 UT, and the He I emission peaks one CDS time resolution element (9.81 s) later, at 22:46:53.0 UT.

Since all the preflare line intensities observed by CDS remain nearly constant for about 4 to 5 minutes after the start of the GOES M1.6 flare at 22:40 UT, the EUV emission observed during the FLT appears to be independent of the GOES event. However, a comparison with the RHESSI 25 - $50 \mathrm{keV}$ light curve (Fig. 2d) reveals correspondences between the peaks in the EUV emission and subpeaks in the hard X-rays. (The HXR light curve covers a shorter interval than the EUV ones due to RHESSF's Earth orbit.) A broad peak in the RHESSI light 
curve from 22:43 to 22:44 UT is followed by the gradual rise in Fe XIX emission that began at 22:43:46.6 UT. The small but impulsive HXR peak at 22:46 UT immediately precedes the impulsive rise of all of the EUV emission lines (also Fig. 3). The broad HXR peak from 22:52 to 22:55 UT corresponds to the second EUV peak seen in Fe XIX, He I, and O V.

A contour map of the RHESSI 20 - $40 \mathrm{keV}$ emission from 22:44:40 to 22:47:20 UT is superimposed on the EIT image at 22:36 UT in Fig. 1(c). This time period includes a plateau in the RHESSI $25-50 \mathrm{keV}$ light curve around the small impulsive peak at 22:46 UT. The contours are 5, 10, 20,40, and $80 \%$ of the peak emission. The spatial resolution is 7 arcsec. The image was obtained using Pixon, a maximum entropy image reconstruction method that attempts to provide the minimum source structure consistent with the RHESSI data (Hurford et al. 2002). It is apparent that there is no X-ray source at the location of the FLT. RHESSI images obtained in other energy bands, at other times and time resolutions, and using the Clean image reconstruction method also showed no source at this location. RHESST's dynamic range is estimated to be 10 to 1 , so this indicates that the HXR emission from the FLT is at most one-tenth of the maximum value in the region.

In Figure 3 we show EUV light curves and Doppler velocity plots for an 8 minute time interval around the FLT's impulsive peak. Doppler velocities for He I, O V, and Si XII were calculated relative to pre-FLT reference wavelengths derived by averaging the fitted centroid wavelengths from the broadened gaussian profile fits in each time element during the 8.8-minute time interval between 22:36:05 and 22:44:55 UT. The associated $1 \sigma$ (scatter) uncertainties were obtained from the same wavelength arrays. For Fe XIX we use measurements during the 2.9-minute time interval between 22:57:01 and 22:59:58 UT late during the decline of the Fe XIX emission. During the FLT's impulsive phase we measure maximum downflow speeds of $28.6 \pm 1.8 \mathrm{~km} \mathrm{~s}^{-1}$ with He I and $32.3 \pm 5.0 \mathrm{~km} \mathrm{~s}^{-1}$ with O V; we also measure maximum upflow speeds of $-21.9 \pm 6.1 \mathrm{~km} \mathrm{~s}^{-1}$ with Si XII and $\sim-150 \mathrm{~km} \mathrm{~s}^{-1}$ with Fe XIX. The largest upflows measured in Fe XIX occur early during the impulsive rise, when the Fe XIX emission is especially weak and yields large uncertainties on the associated velocity measurements; more reliable upflows approaching $-80 \pm 30 \mathrm{~km} \mathrm{~s}^{-1}$ are measured with Fe XIX shortly after the He I and O V intensity peaks. Although not shown here, Fe XIX continues to show upflows $\sim-100 \mathrm{~km} \mathrm{~s}^{-1}$ during the 2 nd Fe XIX peak (3rd HXR peak), during which velocities in the other EUV lines are much smaller than their earlier values.

\section{Discussion and Conclusions}

In their hydrodynamic simulations of the response of a flare loop atmosphere to thicktarget electron heating, Fisher et al. (1985a) found an energy flux threshold $\sim 10^{10}$ ergs 
$\mathrm{cm}^{-2} \mathrm{~s}^{-1}$ above which chromospheric evaporation is "explosive" and below which it is comparatively "gentle." Their velocity calculations revealed that the direction of flow in the transition region $\left(\sim 10^{5} \mathrm{~K}\right)$ is upward $\sim 20 \mathrm{~km} \mathrm{~s}^{-1}$ for gentle evaporation, and downward $\sim 40 \mathrm{~km} \mathrm{~s}^{-1}$ for explosive evaporation (Fisher et al. 1985b). Because all of the EUV emission lines used in the present work were observed simultaneously with a single spectrometer, coalignment of emission from the chromosphere (He I) through the transition region (O V) to the corona (Si XII) and hotter plasma (Fe XIX) is automatic: no co-registration among multiple instruments is required. Then, since upflows in the hot (Si XII and Fe XIX) lines are observed simultaneously and cospatially with downflows in the chromospheric (He I) and transition region $(\mathrm{O} V)$ lines, we conclude not only that chromospheric evaporation occurs during the flare-like transient, but also that the evaporation is "explosive."

Allred et al. (2005) provide radiative hydrodynamic simulations of moderate (electron beam energy flux of $10^{10} \mathrm{ergs}^{-2} \mathrm{~s}^{-1}$ ) and strong $\left(10^{11}\right)$ solar flares. In both cases a relatively gentle phase preceded an explosive phase, with the gentle phase lasting longer in the case of the weaker beam flux. It is possible that the FLT's brief precursor seen with CDS (Figures $2 \& 3$ ) corresponds to this gentle phase. Interestingly, however, in their PBEAM studies of GOES M6.3 and M2.3 flares, Brosius (2003) and Brosius \& Phillips (2004) observed longer precursors ( $\gtrsim 5$ minutes) than the one preceding the FLT.

We put an upper limit on the power in the electron beam at the FLT by computing the beam power required to produce the observed nonthermal HXR emission (e.g., Holman et al. 2003, Sui et al. 2005). We fit the photon spectrum at 22:45:40 - 22:46:20 UT, the time interval of the small impulsive peak, with a model consisting of bremsstrahlung from isothermal plasma and thick-target bremsstrahlung from a power-law electron distribution with a low-energy cutoff. The best fit gave a spectral index of -7.4, a low-energy cutoff of $17.5 \mathrm{keV}$, and a beam power of $1.1 \times 10^{28} \mathrm{erg} \mathrm{s}^{-1}$. The value of $\chi^{2}$ was found to increase by one if the low-energy cutoff increased to $21 \mathrm{keV}$, decreasing the beam power to $3.6 \times 10^{27}$ $\operatorname{erg~s}^{-1}$, or if it decreased to $15 \mathrm{keV}$, increasing the beam power to $3.0 \times 10^{28} \mathrm{erg} \mathrm{s}^{-1}$. The power at the FLT must have been at least an order of magnitude less than this, based on RHESST's dynamic range. Taking the source area to be comparable to the CDS pixel size of $80 \operatorname{arcsec}^{2}$ and using one-tenth the computed powers, the corresponding beam energy flux at the FLT is $0.86-7.2 \times 10^{9} \mathrm{erg} \mathrm{cm}^{-2} \mathrm{~s}^{-1}$, with a most probable value of $2.5 \times 10^{9} \mathrm{erg} \mathrm{cm}^{-2}$ $\mathrm{s}^{-1}$. If an energy flux of $10^{10} \mathrm{erg} \mathrm{cm}^{-2} \mathrm{~s}^{-1}$ or greater is required for explosive evaporation, we conclude that the beam area must have been smaller than the CDS pixel size.

We speculate that the slow rise of the Fe XIX emission during the earliest HXR peak in our observations may be due to direct coronal heating by the same (magnetic reconnection) process that accelerates the nonthermal electrons that later impact the chromosphere and 
cause evaporation. This is supported by simultaneous observations of no rapid intensity enhancements in the cool lines, which indicates that the chromosphere was not involved during this stage. Wang et al. (2006) observed a number of small "flarelike" brightenings above the limb in Fe XIX emission at $1118 \AA$ with SOHO's SUMER (the most intense of which were associated with GOES events). The average duration of the brightenings was 25 minutes, comparable to the 18 minute duration of the Fe XIX emission from our FLT. The SUMER brightenings may be off-limb manifestations of events like the FLT reported here.

Both the maximum and the time-integrated Fe XIX intensities in the FLT are two orders of magnitude smaller than their counterparts in the GOES M2.3 flare analyzed by Brosius \& Phillips (2004). From this we estimate that the FLT is weaker than a GOES C-class flare. The fact that we are able to observe and analyze such a weak event suggests that spectroscopic studies of smaller, weaker transients can be pursued with SOHO's CDS, the Extreme Ultraviolet Normal Incidence Spectrograph (EUNIS; Brosius, Rabin, \& Thomas 2007), and Hinode's Extreme-ultraviolet Imaging Spectrometer (EIS), along with RHESSI. 


\section{REFERENCES}

Allred, J. C., Hawley, S. L., Abbett, W. P., \& Carlsson, M. 2005, ApJ, 630, 573

Antonucci, E., et al. 1999, "Flare Dynamics," in The Many Faces of the Sun (SpringerVerlag: New York), ed. K. T. Strong, J. L. R. Saba, B. M. Haisch, \& J. T. Schmelz, p. 331

Bornmann, P. L. 1999, "Chromospheric Evaporation Theory," in The Many Faces of the Sun (Springer-Verlag: New York), ed. K. T. Strong, J. L. R. Saba, B. M. Haisch, \& J. T. Schmelz, p. 301

Brosius, J. W. 2001, ApJ, 555, 435

Brosius, J. W. 2003, ApJ, 586, 1417

Brosius, J. W., \& Phillips, K. J. H. 2004, ApJ, 613, 580

Brosius, J. W., Rabin, D. M., \& Thomas, R. J. 2007, ApJ, 656, L41

Czaykowska, A., Alexander, D., \& De Pontieu, B. 2001, ApJ, 552, 849

Czaykowska, A., De Pontieu, B., Alexander, D., \& Rank, G. 1999, ApJ, 521, L75

Delaboudinière, J.-P., et al. 1995, Sol. Phys., 162, 291

Emslie, A. G., Li, P., \& Mariska, J. T. 1992, ApJ, 399, 714

Falchi, A., Teriaca, L., \& Maltagliati, L. 2006, Sol. Phys., 239, 193

Fisher, G. H., Canfield, R. C., \& McClymont, A. N. 1985a, ApJ, 289, 414

Fisher, G. H., Canfield, R. C., \& McClymont, A. N. 1985b, ApJ, 289, 425

Fisher, G. H., Canfield, R. C., \& McClymont, A. N. 1985c, ApJ, 289, 434

Harrison, R. A., et al. 1995, Sol. Phys., 162, 233

Holman, G. D., Sui, L., Schwartz, R. A., \& Emslie, A. G. 2003, ApJ, 595, L97

Hurford, G. J., Schmahl, E. J., Schwartz, R. A., et al. 2002, Sol. Phys., 210, 61

Lin, R. P., et al. 2002, Sol. Phys., 210, 3

Mazzotta, P., Mazzitelli, G., Colafrancesco, S., \& Vittorio, N. 1998, A\&AS, 133, 403

Milligan, R. O., Gallagher, P. T., Mathioudakis, M., Bloomfield, D. S., Keenan, F. P., \& Schwartz, R. A. 2006a, ApJ, 638, L117

Milligan, R. O., Gallagher, P. T., Mathioudakis, M., \& Keenan, F. P. 2006b, ApJ, 642, L169

Neupert, W. M. 1968, ApJ, 153, L59

Pike, C. D., \& Mason, H. E. 2002, Sol. Phys., 206, 359 
Smith, D. M., et al. 2002, Sol. Phys., 210, 33

Sui, L., Holman, G. D., \& Dennis, B. R. 2005, ApJ, 626, 1102

Teriaca, L., Falchi, A., Cauzzi, G., Falciani, R., Smaldone, L. A., \& Andretta, V. 2003, ApJ, 588,596

Teriaca, L., Falchi, A., Falciani, R., Cauzzi, G., \& Maltagliati, L. 2006, A\&A, 455, 1123

Thompson, W. T., 1999, CDS Software Note 53, http://solg2.bnsc.rl.ac.uk/software/notes.shtml

Wang, T. J., Innes, D. E., \& Solanki, S. K. 2006, A\&A, 455, 1105

This preprint was prepared with the AAS IATEX macros v5.2. 

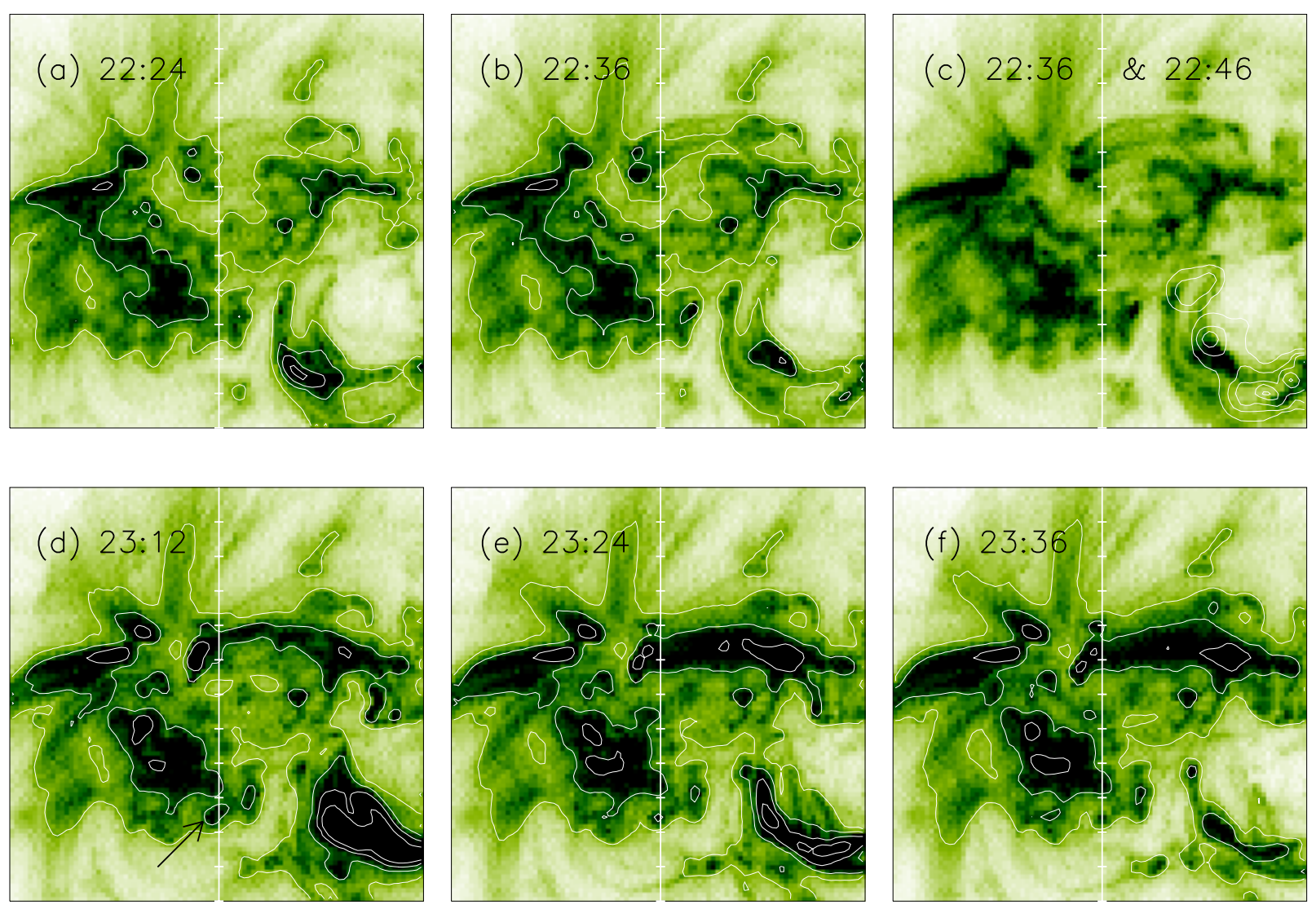

Fig. 1. - Sequence of $4^{\prime} \times 4^{\prime}$ EIT $195 \AA$ images obtained between 22:24 and 23:36 UT on 2004 July 22, showing the location of the $4 \operatorname{arcsec} \times 4 \operatorname{arcmin}$ CDS slit (centered at $\mathrm{x}=-141^{\prime \prime}$, $\mathrm{y}=+58^{\prime \prime}$ ) within AR 10652. All six frames are normalized to the same negative intensity scale and, except for frame (c), include the same intensity contours (which represent successive intensity increases by factors of 2). Contours in frame (c) correspond to 5, 10, 20, 40, and $80 \%$ of the maximum 20-40 keV RHESSI HXR count rate at 22:46 UT. The times at which the images were obtained are given in the upper left corner of each frame. Solar north is up and west is to the right. Horizontal tick marks indicate the endpoints of the twelve $4^{\prime \prime} \times 20^{\prime \prime}$ spatial pixels into which the CDS spectra were compressed. The FLT described and analyzed in this work occurred in the 3rd CDS slit pixel up from the bottom (centered at $\mathrm{x}=-141^{\prime \prime}, \mathrm{y}=-12^{\prime \prime}$ ), indicated with an arrow at 23:12 UT and outlined by the 2nd EIT contour in frames (d) and (e). Notice in frame (c) that no RHESSI source appears at this location (see text). 

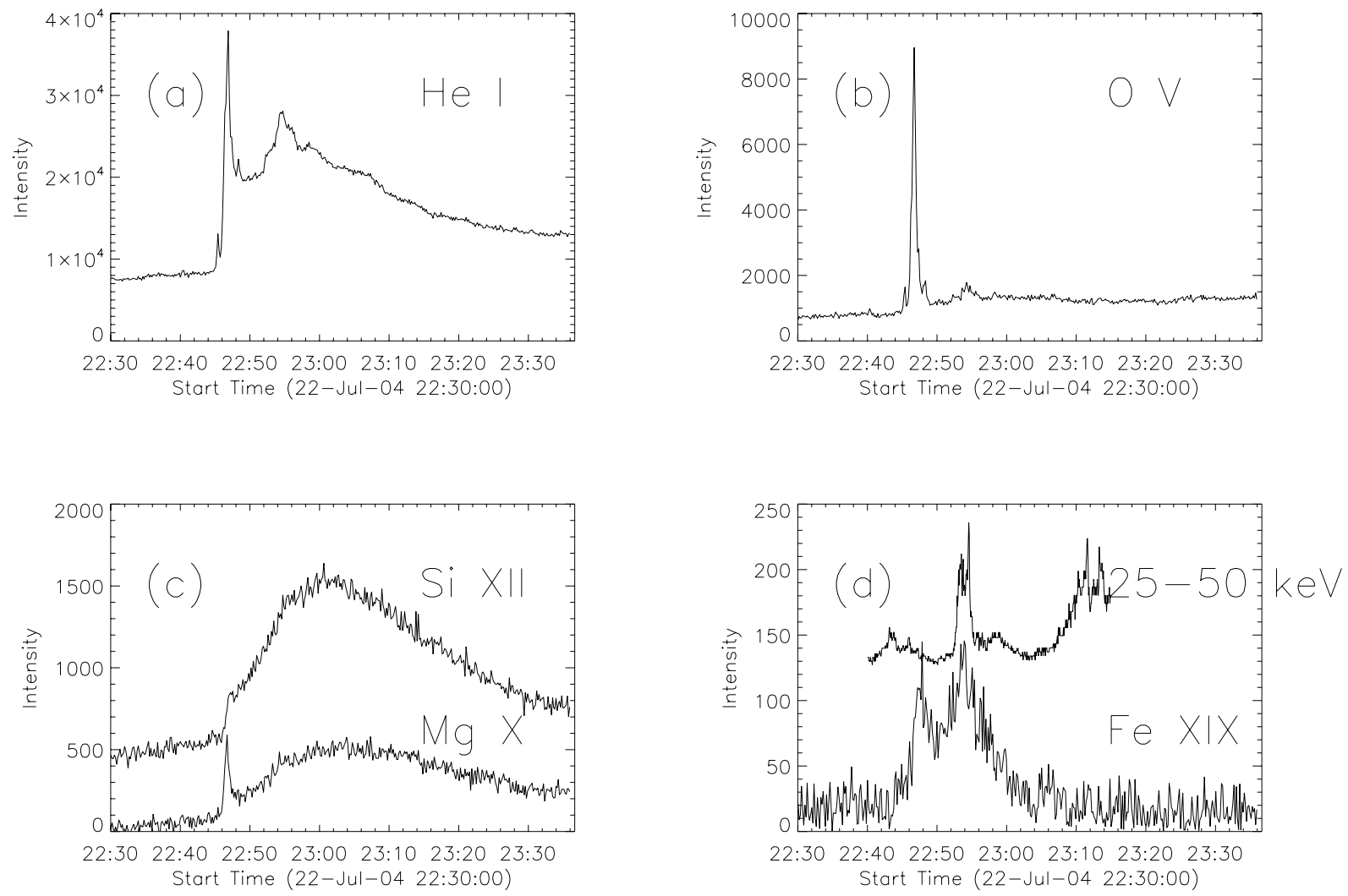

Fig. 2. - Integrated line intensities in $\mathrm{erg}^{\mathrm{cm}} \mathrm{cm}^{-2} \mathrm{~s}^{-1} \mathrm{sr}^{-1}$ for (a) He I at $584.3 \AA$, (b) O V at $629.7 \AA$, (c) Mg X at $624.9 \AA$ (shifted downward by $300 \mathrm{erg} \mathrm{cm}^{-2} \mathrm{~s}^{-1} \mathrm{sr}^{-1}$ ) with Si XII at $520.7 \AA$, and (d) Fe XIX at $592.2 \AA$ as functions of time UT measured with SOHO's CDS, obtained from the 3rd slit pixel up from the bottom (see arrow in Fig. 1d). Frame (d) above also shows the 25-50 keV HXR light curve obtained with RHESSI, in arbitrary units. The pair of weak HXR peaks, centered around 22:43:20 and 22:46:00 UT, precedes the impulsive rise of the EUV lines. A third (broader and stronger) HXR peak, centered around 22:54:00 UT, is associated with secondary peaks in the EUV emission. In this work we focus on the initial impulsive rise, displayed over a shorter time interval in Figure 3. 

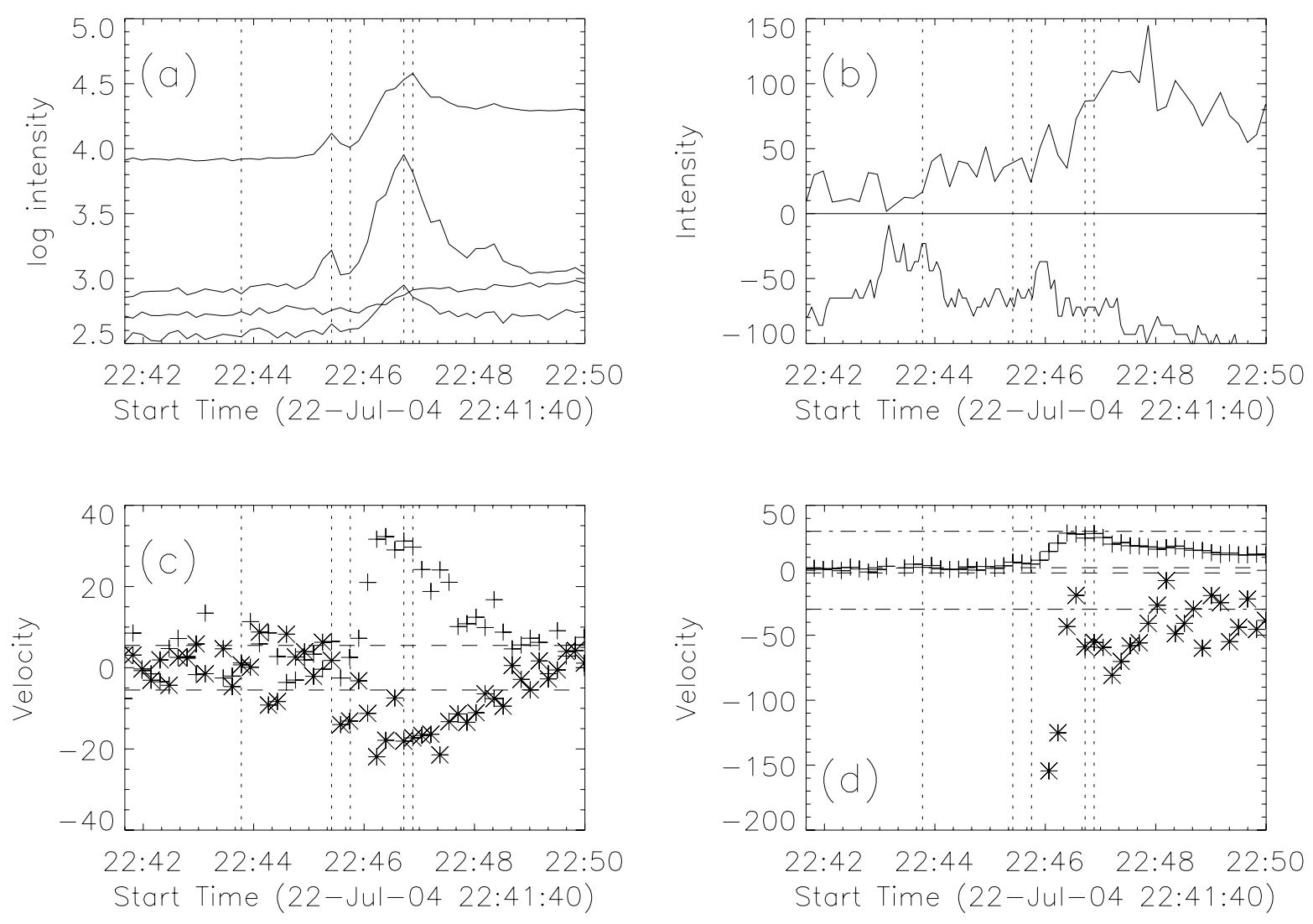

Fig. 3.- Light curves and relative Doppler velocities during the impulsive rise of the FLT. Frame (a) displays the log of the integrated line intensity (in erg $\mathrm{cm}^{-2} \mathrm{~s}^{-1} \mathrm{sr}^{-1}$ ) for the He I line at $584.3 \AA$ (top), $\mathrm{O} \mathrm{V}$ at $629.7 \AA$, Si XII at $520.7 \AA$, and Mg X at $624.9 \AA$ (bottom). Frame (b) displays the integrated line intensity for Fe XIX at $592.2 \AA$ (top) and the 25-50 $\mathrm{keV}$ HXR count rate (bottom, arbitrary units). Dotted vertical lines indicate the beginning of a slow, steady rise of the Fe XIX intensity at 22:43:46.6 UT, a precursor peak seen in He $\mathrm{I}, \mathrm{O} \mathrm{V}$, and $\mathrm{Mg} \mathrm{X}$ at 22:45:24.7 UT, the beginning of the He I and $\mathrm{O} \mathrm{V}$ impulsive rise at 22:45:45.0 UT, the impulsive peak seen in O V (and O III and Mg X) at 22:46:43.2 UT, and the impulsive peak seen in He I (and He II) at 22:46:53.0 UT. Frame (c) displays relative Doppler velocities (in $\mathrm{km} \mathrm{s}^{-1}$ ) seen in $\mathrm{O} \mathrm{V}$ (plus signs) and Si XII (asterisks), and (d) shows relative Doppler velocities in He I (plus signs) and Fe XIX (asterisks). Negative velocities correspond to upflows (blueshifts) and positive to downflows (redshifts). Dashed horizontal lines indicate uncertainties $\sim 5 \mathrm{~km} \mathrm{~s}^{-1}$ on the reference wavelengths of $\mathrm{O} \mathrm{V}$ and $\mathrm{Si}$ XII, and $\sim 2 \mathrm{~km} \mathrm{~s}^{-1}$ for He I; dot-dashed horizontal lines indicate uncertainties $\sim 30 \mathrm{~km} \mathrm{~s}^{-1}$ for Fe XIX. 